\title{
Corrigendum
}

\section{Whiteness and Masculinity in Richard Lou's ReCovering Memphis: ReContexting Bodies - CORRIGENDUM}

\section{JODY STOKES-CASEY}

https://doi.org/I0.1017/S0021875818001470, Published by Cambridge University Press, 8 January 2019

When originally published, the article included the incorrect email address for Jody Stokes-Casey ${ }^{1}$. This has been corrected in the original online version.

' Jody Stokes-Casey, "Whiteness and Masculinity in Richard Lou's ReCovering Memphis: ReContexting Bodies", Journal of American Studies, (Cambridge University Press, https://doi.org/10.1017/S0021875818001470). 